\title{
Cross-check of different techniques for two-dimensional power spectral density measurements of $x$-ray optics
}

\author{
Valeriy V. Yashchuk, Steve C. Irick, Eric M. Gullikson, Malcolm R. Howells, \\ Alastair A. MacDowell, Wayne R. McKinney, Farhad Salmassi, Tony Warwick \\ Lawrence Berkeley National Laboratory, 1 Cyclotron Road, Berkeley, CA, USA 94720
}

\begin{abstract}
The consistency of different instruments and methods for measuring two-dimensional (2D) power spectral density (PSD) distributions are investigated. The instruments are an interferometric microscope, an atomic force microscope (AFM) and the X-ray Reflectivity and Scattering experimental facility, all available at Lawrence Berkeley National Laboratory. The measurements were performed with a gold-coated mirror with a highly polished stainless steel substrate. It was shown that these three techniques provide essentially consistent results. For the stainless steel mirror, an envelope over all measured PSD distributions can be described with an inverse power-law PSD function. It is also shown that the measurements can be corrected for the specific spatial frequency dependent systematic errors of the instruments. The AFM and the X-ray scattering measurements were used to determine the modulation transfer function of the interferometric microscope. The corresponding correction procedure is discussed in detail. Lower frequency investigation of the 2D PSD distribution was also performed with a long trace profiler and a ZYGO GPI interferometer. These measurements are in some contradiction, suggesting that the reliability of the measurements has to be confirmed with additional investigation. Based on the crosscheck of the performance of all used methods, we discuss the ways for improving the 2D PSD characterization of X-ray optics.
\end{abstract}

Keywords: interferometric microscope, atomic force microscope, X-ray scattering, power spectral density, long trace profiler, interferometer, X-ray optics, optical metrology

\section{INTRODUCTION}

The task of designing high performance X-ray optical systems ${ }^{1}$ requires the development of sophisticated X-ray scattering calculations based on rigorous information about the optics. One of the most insightful approaches to these calculations is based on the two-dimensional (2D) power spectral density (PSD) distribution of the surface height, allowing for the evaluation of three-dimensional distributions of X-rays scattered by the optics. ${ }^{2,3}$ A comprehensive discussion of the importance of characterization of highly finished optical surfaces via the PSD distribution can be found in Refs. 4 and 5.

The 2D PSD function $S_{2}(u, v)$ may be viewed as a Fourier decomposition of the 2D surface height distribution $h(x, y)$ into harmonic basis functions: ${ }^{6,7}$

$$
S_{2}(u, v)=\lim _{A \rightarrow \infty}\left\langle\frac{1}{A}\left|\int_{-L_{y} / 2}^{L_{y} / 2} d y \int_{-L_{x} / 2}^{L_{x} / 2} h(x, y) e^{-2 \pi i(u x+v y)} d x\right|^{2}\right\rangle,
$$

where $L_{x}$ and $L_{y}$ are the tangential and sagittal dimensions of the measured surface region, $A=L_{x} L_{y} ; u$ and $v$ are the spatial frequency variables corresponding to the tangential, $x$, and sagittal, $y$, coordinates. In the case of discreet measurements with pixel dimensions $\Delta x$ and $\Delta y, \mathrm{M}$ and $\mathrm{N}$ pixels in the tangential and sagittal directions, respectively, the 2D PSD distribution can be evaluated from the height distribution $h_{m, n}$ via equation

$$
S_{2}(l, k)=M N \Delta x \Delta y\left|F_{l, k}\right|^{2},
$$


where $F_{l, k}$ are the elements of the Fourier transform matrix,

$$
F_{l, k}=\frac{1}{M} \sum_{m=0}^{M-1}\left[\exp \left(\frac{-2 \pi i m l}{M}\right) \frac{1}{N} \sum_{n=0}^{N-1} h_{m, n} \exp \left(\frac{-2 \pi i n k}{N}\right)\right] .
$$

The corresponding estimates of the tangential and sagittal one-dimensional (1D) two-sided PSDs $S_{1}^{\prime}(l)$ and $S_{1}^{\prime}(k)$ can be obtained by summing over rows ( $l$ ) or columns $(k)$, respectively. Here $0 \leq l \leq M-1$ and $0 \leq k \leq N-1$, and prime signifies a two-sided PSD. These are then converted to one-sided (positive frequency only) just like the ones calculated from lines on the surface directly

$$
S_{1}(l)=2 S_{1}^{\prime}(l) g(l) \text { and } S_{1}(k)=2 S_{1}^{\prime}(k) g(k)
$$

where $0 \leq l \leq M / 2,0 \leq k \leq N / 2 ; g(l)=1 / 2$ at $l=0, M / 2, g(k)=1 / 2$ at $k=0, N / 2$, and $g(l)=1$ and $g(k)=1$ otherwise.

In this work, we are investigating the consistency of different instruments and methods for measuring 2D PSD distributions. The instruments are the Micromap-570 interferometric microscope ${ }^{8}$ (IM), the Digital Instruments Dimension 3100 atomic force microscope (AFM), ${ }^{9}$ and the X-ray Reflectometry and Scattering (XRS) experimental facility ${ }^{10}$ all available at Lawrence Berkeley National Laboratory. The measurements were performed using a mirror with gold coating on a highly polished stainless steel substrate. ${ }^{11}$ For the mirror, the surface finish was found to be essentially isotropic, allowing straightforward comparison of the one dimensional (1D) PSD spectra obtained by convolution of the 2D PSD distributions measured with the IM (Section 2) and the AFM (Section 3) and the 1D PSD spectra extracted from the XRS experiment (Section 4). The main conclusion from the crosscheck (Section 5) is that all three techniques provide essentially consistent results. At spatial frequencies from $\sim 0.1 \mu \mathrm{m}^{-1}$ to $50 \mu \mathrm{m}^{-1}$, the X-ray scattering measurements agree reasonably well with the AFM measurements. The frequency range available for the interferometric microscope measurement is shifted to the lower frequencies, $0.001 \mu \mathrm{m}^{-1}-2 \mu \mathrm{m}^{-1}$; but still in the overlapped range, we observed reasonable agreement between the PSD magnitudes measured with these three different instruments.

A straightforward transformation of the area distribution of the residual surface heights available from the IM or AFM measurement into a 2D PSD distribution generally provides spectra with distortion caused by an unknown spatial frequency response of the instrument. The response is characterized with the modulation transfer function (MTF), determining the bandwidth of the instrument. ${ }^{12}$ The MTF contains contributions from the instrument optical system, detector, signal processing, software algorithm, and environmental factors. Generally, these contributions are difficult to account for separately. The instrument MTF can be evaluated by comparing a PSD distribution measured using a known test surface with a corresponding ideal numerically simulated PSD. ${ }^{13,14}$ The square root of the ratio of the measured and simulated PSD distributions gives the MTF of the instrument. There is another, totally experimental, way to estimate the MTF, where the same surface is measured with different techniques with overlapping spatial frequency ranges. In the present work, we employ the PSD measurements of a mirror performed with the atomic force microscope and evaluated from mirror surface scattering and reflectivity measured with the XRS facility in order to estimate the MTF of the Micromap interferometric microscope (Section 6). Lower frequency investigation of 2D PSD distribution was also performed with a long trace profiler ${ }^{15}$ (LTP) and a ZYGO GPI interferometer ${ }^{16}$ (Section 7). These measurements are in some contradiction, suggesting that the reliability of the measurements has to be confirmed with additional investigation.

\section{2D PSD MEASUREMENTS WITH INTERFEROMETRIC MICROSCOPE}

In the Optical Metrology Laboratory (OML) at the Advanced Light Source (ALS), LBNL, the Micromap-570 ${ }^{8}$ interferometric microscope is a basic metrology tool for testing of the surface finish of X-ray optics with sub-Angstrom rms roughness. The standard list of output parameters of the IM measurement includes values of roughness averaged over an area and along a sample line. In order to exclude contributions from the imperfections of the IM optics, a 
surface height distribution is measured in the super smooth mode. In this mode, an output height distribution is the result of two consecutive smooth phase mode measurements over two different surface areas. The measured height distributions are subtracted one from another and renormalized with factor $2^{1 / 2}$ in order to preserve the rms roughness value. In the Micromap measurement presented throughout this work, the contribution due to the instrument noise was found to be negligibly small.

In order to transform the area distribution of the residual surface heights available from the measurement with the Micromap into a 2D PSD distribution of the surface height, a dedicated procedure and software program have been developed. ${ }^{17}$ With this procedure, a $2 \mathrm{D}$ height distribution measured with the Micromap-570 is first detrended with a surface determined by best-fit 2D toroidal surface. The general expression for the surface is given by the expression:

$$
S(x, y)=k_{00}+k_{10} x+k_{20} x^{2}+k_{01} y+k_{02} y^{2}+k_{11} x y+k_{21} x^{2} y+k_{12} x y^{2}+k_{22} x^{2} y^{2} .
$$

The procedure also incorporates correction of one of the spectral distortions of the PSD measurement with the Micromap caused by the asymmetry of the read-out process of the instrument's CCD camera. The effectiveness of the developed procedure has been demonstrated in Ref. 17 with a number of PSD measurements with different X-ray optics including mirrors and a grating.

Figure 1 presents PSD spectra for the Micromap measurements of the stainless steel mirror. The 2D PSD distributions measured with all available objectives, $2.5 \times, 5 \times, 10 \times, 20 \times$, and $50 \times$, were processed with the correction procedure $^{17}$. The PSD measurements with different objectives allow for extending the available spatial frequency range $4 \cdot 10^{-4} \mu \mathrm{m}^{-1}-2 \mu \mathrm{m}^{-1}$. The higher spatial frequency roll-off observed for an objective with lower resolution can be corrected with the measurement with a higher resolution objective. The high frequency roll-off systematically observed with all objectives is a manifestation for the instrument MTF.

A noticeable feature of the data in Fig. 1 is the similarity of the tangential and sagittal PSD spectra. Although the individual measurements do not generally show exact similarity of the tangential and sagittal spectra, the overall envelopes over the tangential (Fig. 1a) and the sagittal (Fig. 1b) spectra are essentially identical, suggesting a high degree of isotropy for the mirror surface finish.
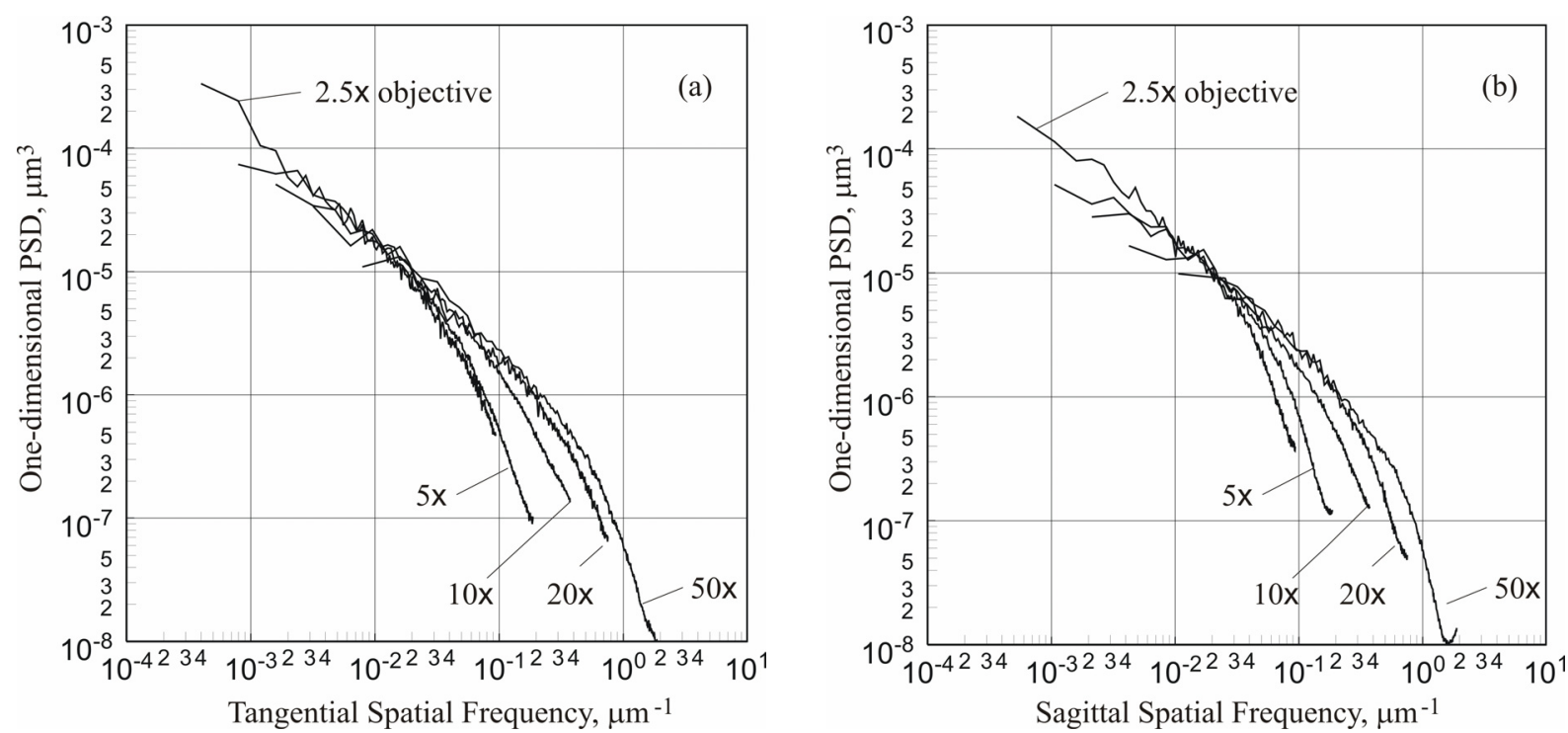

Figure 1: $a$ - Tangential and $b$ - sagittal 1D PSD spectra extracted from the Micromap measurement with different objectives. In spite of the fact that individual measurements do not generally show exact similarity of the tangential and sagittal spectra, the overall envelopes over the tangential and the sagittal spectra are significantly identical, suggesting the high degree of isotropy for the mirror surface finish. 
The spectra shown in Fig. 1 should be compared with similar measurements performed using a silicon mirror with a platinum coating and surface rms roughness of approximately $3 \AA$ and presented in Fig. 6 of Ref. 17. This mirror was cylindrically shaped in the sagittal direction with a radius of curvature of approximately $24.9 \mathrm{~cm}$. Such a mirror figure renders measurement difficult at low magnification because the steep curvature in the sagittal direction reduces the field of view that is in focus. The numerical aperture is therefore restricted in the sagittal plane resulting in greater roll-off to higher frequencies for the sagittal case even for an isotropic surface finish. This problem does not appear with a flat mirror, as used in this present work.

\section{2D PSD MEASUREMENTS WITH ATOMIC FORCE MICROSCOPE}

The stainless steel mirror surface finish was investigated with an atomic force microscope at the Center for X-Ray Optic's Nano-Fabrication facility, LBNL. A series of AFM scans were performed using a Digital Instruments 3100 machine with NanoScope software. ${ }^{9}$ The AFM provides surface height distributions, which can be converted with the built-in software into 1D and 2D PSD spectra. The instrument allows PSD measurements for the spatial frequency range from approximately $0.01 \mu \mathrm{m}^{-1}$ to $50 \mu^{-1}$, which corresponds to a measuring surface area of up to $100 \times 100 \mu \mathrm{m}^{2}$ with $512 \times 512$ elements.

The AFM instrument used in tapping mode collects data by resonating an atomically sharp tip at the end of a cantilever over a desired surface. Ordinary etched silicon cantilever tips with $15 \mathrm{~nm}$ to $20 \mathrm{~nm}$ tip diameters were used with response frequencies of approximately $290 \mathrm{kHz}$. The scans ranged from 5 micrometers to 100 micrometers on a side. To remove AFM trends and artifacts after measurement, each scan was put through two modification steps. To remove any tilt, bow, or S-shapes from the overall image, a Plane-fit program within the NanoScope software was used. The software generates a single $1^{\text {st }}, 2^{\text {nd }}$ or $3^{\text {rd }}$ order polynomial fit for the entire image and then subtracts that polynomial from the image. A $3^{\text {rd }}$ order Plane-fit removes any tilt, bow, and S-shapes from the image and this procedure was used on all images. A zero order flatten routine was next used to remove unwanted features from the individual scan lines. The flatten routine within the NanoScope software uses all unmasked portions of the scan to calculate individual least-squares fit polynomials. The polynomials are subtracted from scan lines individually. This is useful for surfaces that have sporadic, tall features in predominantly flat areas such as the surface of the mirror under investigation. The zero order removes the $\mathrm{Z}$ offset between scan lines by subtracting the average $\mathrm{Z}$ value of the selected segment from every point in the scan line.

Figure 2 presents a central part of the 2D PSD distribution measured with the AFM over $5 \times 5 \mu^{2}$ area with $512 \times 512$ elements. The measurement covers the spatial frequency range from $0.2 \mu \mathrm{m}^{-1}$ to about $50 \mu \mathrm{m}^{-1}$. The azimuth symmetry of the distribution suggests an isotropic surface finish of the mirror, which was also found for the lower spatial frequency range with the Micromap measurements shown in Fig. 1.

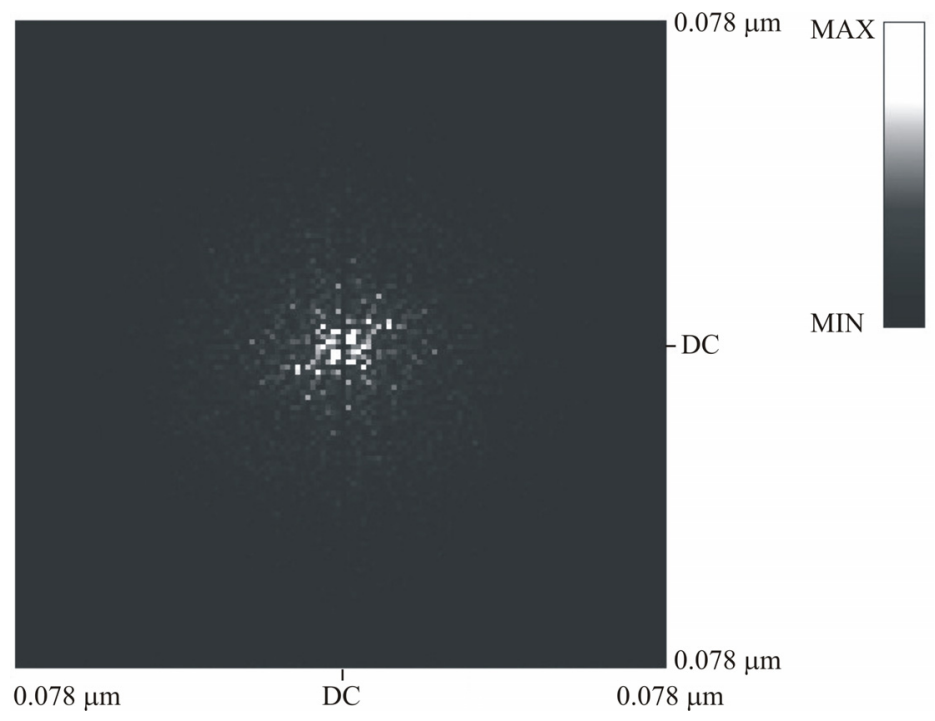

Figure 2: Central part of the 2D PSD distribution measured with the AFM over $5 \times 5 \mu^{2}$ area with $512 \times 512$ elements that corresponds to the spatial frequency range from $0.2 \mu \mathrm{m}^{-1}$ to about $50 \mu \mathrm{m}^{-1}$. The azimuthal symmetry of the distribution suggests an isotropic surface finish of the mirror. (Compare with analogous data for lower spatial frequencies shown in Fig. 1). 
Figure 3 accumulates the 1D PSD spectra measured with the AFM over different areas, covering the whole spatial frequency range available with the AFM. The measurements in Fig. 3 have a high degree of consistency for the range from approximately $1 \mu \mathrm{m}^{-1}$ to $50 \mu \mathrm{m}^{-1}$. At lower frequencies, between about $0.3 \mu \mathrm{m}^{-1}$ and $1 \mu \mathrm{m}^{-1}$, there is a significant spread of the PSD magnitudes obtained in the scans over different areas. Note, that the PSD data for this range are available from the interferometric microscope measurements - Fig. 1, providing a possibility for cross check of the instruments. The lower frequency roll-off systematically seen for all AFM measurements shown in Fig. 3 is due to the detrending procedure discussed above.

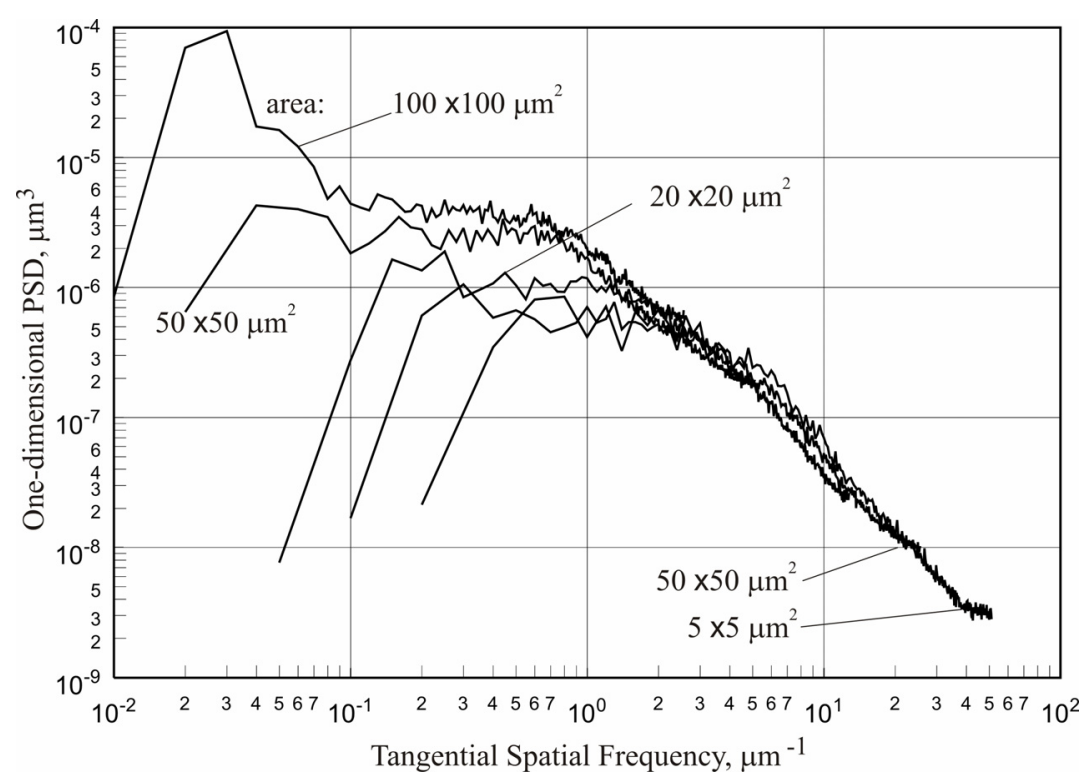

Figure 3: 1D PSD spectra measured with the AFM instrument over different surface areas. For the spatial frequency range above $1 \mu \mathrm{m}^{-1}$, the measurements are very consistent. The spread of the measurements at $\sim 0.3$ $\mu \mathrm{m}^{-1}-1 \mu \mathrm{m}^{-1}$ is probably due to instrumental MTFs, different for different measurement arrangements. The AFM MTF can be evaluated by comparison with the PSD data for this range available from the IM measurements - Fig. 1. The lower frequency roll-off systematically seen for all AFM measurements is due to the detrending procedure discussed in the text.

\section{X-RAY REFLECTIVITY AND SCATTERING EXPERIMENTS}

The X-ray reflectivity and scattering measurements were performed at the CXRO (Center for X-ray Optics) Reflectometry and Scattering experimental facility at the ALS beamline BL6.3.2 ${ }^{10}$. Such measurements can be converted into PSD spectra ${ }^{18,19}$ for the spatial frequency range from $0.1 \mu \mathrm{m}^{-1}$ to $100 \mu^{-1}$ for $100-1000 \mathrm{eV}$ X-rays. This frequency range overlaps at the lower frequencies with the Micromap-570 interferometric microscope measurements, providing a possibility for crosschecking these two PSD measurement techniques. A similar comparative study of the optical surfaces with X-ray scattering and atomic force microscopy has been performed previously (see e.g., Ref. 20) and has demonstrated good quantitative agreement between the techniques.

The reflectance measurement was made in the range of scattering angles of 0-60 degrees. The solid line in Fig. 4 presents the measured dependence. Assuming the surface roughness height distribution obeys a normal distribution, the surface is described by a single parameter of rms roughness equal to the dispersion of an unshifted Gaussian function. This roughness parameter can be estimated from processing the data based on the Born approximation (see e.g., Ref. 3). The dashed line in Fig. 4 corresponds to a distribution calculated assuming a surface roughness of $18 \AA$. The discrepancy seen at larger scattering angles may be due to both the deviation of the roughness height distribution from the normal distribution observed for most of super polished X-ray mirrors, ${ }^{4,17,21}$ and also due to the simplifying assumptions of our theoretical approach. 
The X-ray energy dependence of the reflectance measured at the incidence angle of $1.5^{\circ}$ is shown in Fig. 5. For illustration, the reflectance corresponding to the ideal surface with zero roughness is shown with the dashed line together with the reflectances calculated assuming rms roughness values of $10 \AA, 15 \AA$, and $20 \AA$. The experimental data match a theoretical reflectance calculated assuming the surface roughness of approximately $20 \AA$. Note that the reflectance measurement provides a mirror surface roughness larger than the roughness measured with the Micromap by a factor of approximately 2. However, the Micromap MTF correction described in the following section will remove a significant fraction of this difference. The roughness value also has a strong dependence on the way of calculation and the bandwidth of the measuring set-up, so roughness is not a sufficient characterization of a surface for a conclusive comparison of these two measurement techniques. For a much more informative comparison, we use the PSD spectra.

The PSD spectra were extracted from the scattering measurements made with the X-ray beam at two grazing incidence angles of $1.5^{\circ}$ and $5^{\circ}$ and with photon energy of $92 \mathrm{eV}$. Figure 6 shows the PSD spectra obtained this way.

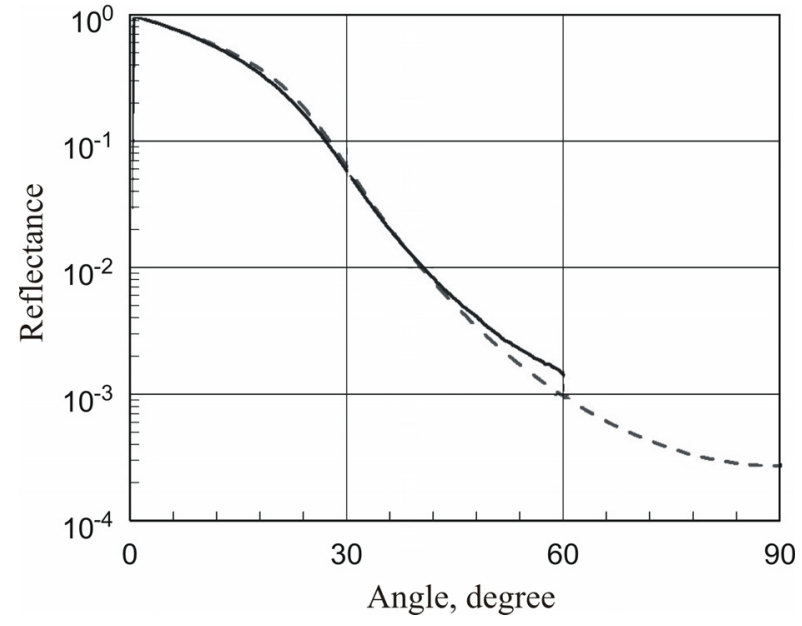

Figure 4: Solid line - X-ray reflectivity angle dependence measured for the stainless steel mirror. The dashed line corresponds to the theoretical curve calculated assuming the mirror surface roughness of approximately $18 \AA$. The measurement was performed at the X-ray energy of $92 \mathrm{eV}$.

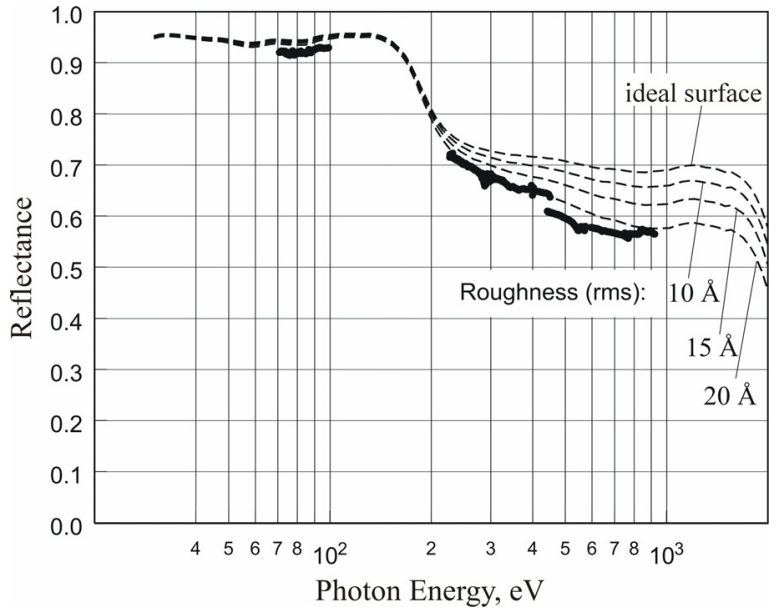

Figure 5: The merged filled circles - X-ray energy dependence of the surface reflectance measured with the stainless steel mirror. The dashed lines correspond to the theoretical spectra calculated assuming the mirror surface roughness of $0 \AA$ (ideal surface), $10 \AA$, $15 \AA$, and $20 \AA$.

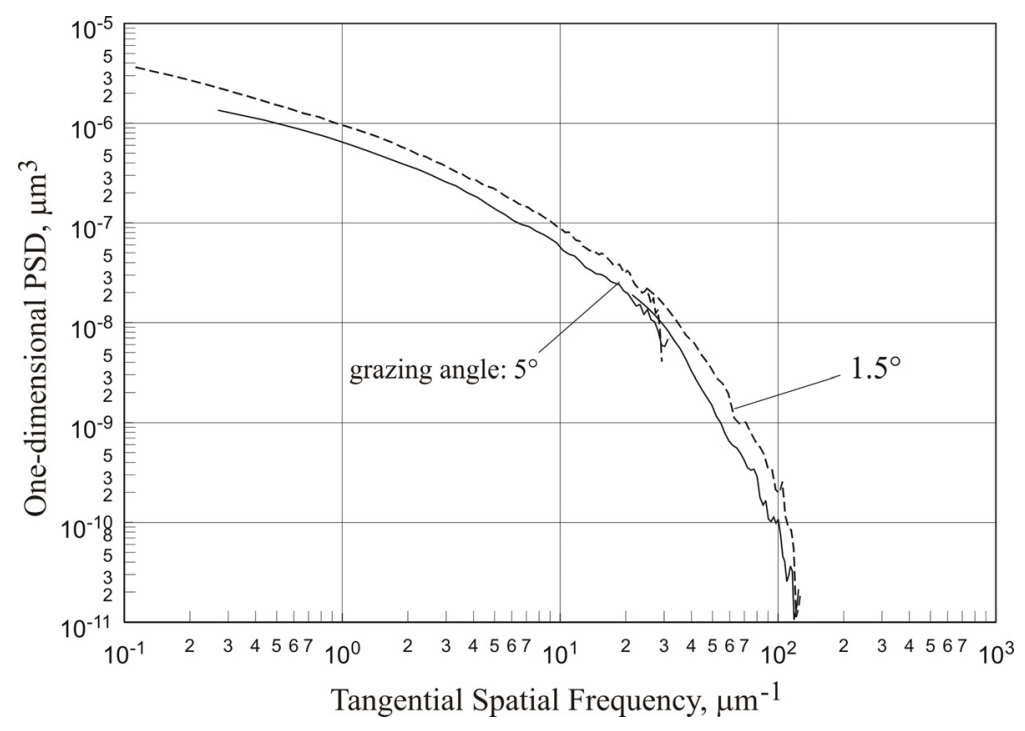

Figure 6: PSD spectra extracted from the X-ray scattering measurements with the stainless steel mirror. The scattering measurements used were made with the Xray beam at two grazing incidence angles of $1.5^{\circ}$ and $5^{\circ}$ and with photon energy of $92 \mathrm{eV}$. The procedure to calculate the spectra assumes an isotropic topography of the surface. 


\section{COMPARISON OF THREE TECHNIQUIES FOR MEASURING PSD DISTRIBUTIONS}

The PSD spectra obtained with three different techniques (Figs. 1a, 3, and 6) are plotted together in Fig. 7. The AFM spatial frequency range almost coincides with the frequency range of the X-ray scattering experiment. It also overlaps at the lower frequencies with the Micromap-570 interferometric microscope measurements. This gives a possibility for crosschecking the PSD measurement techniques.

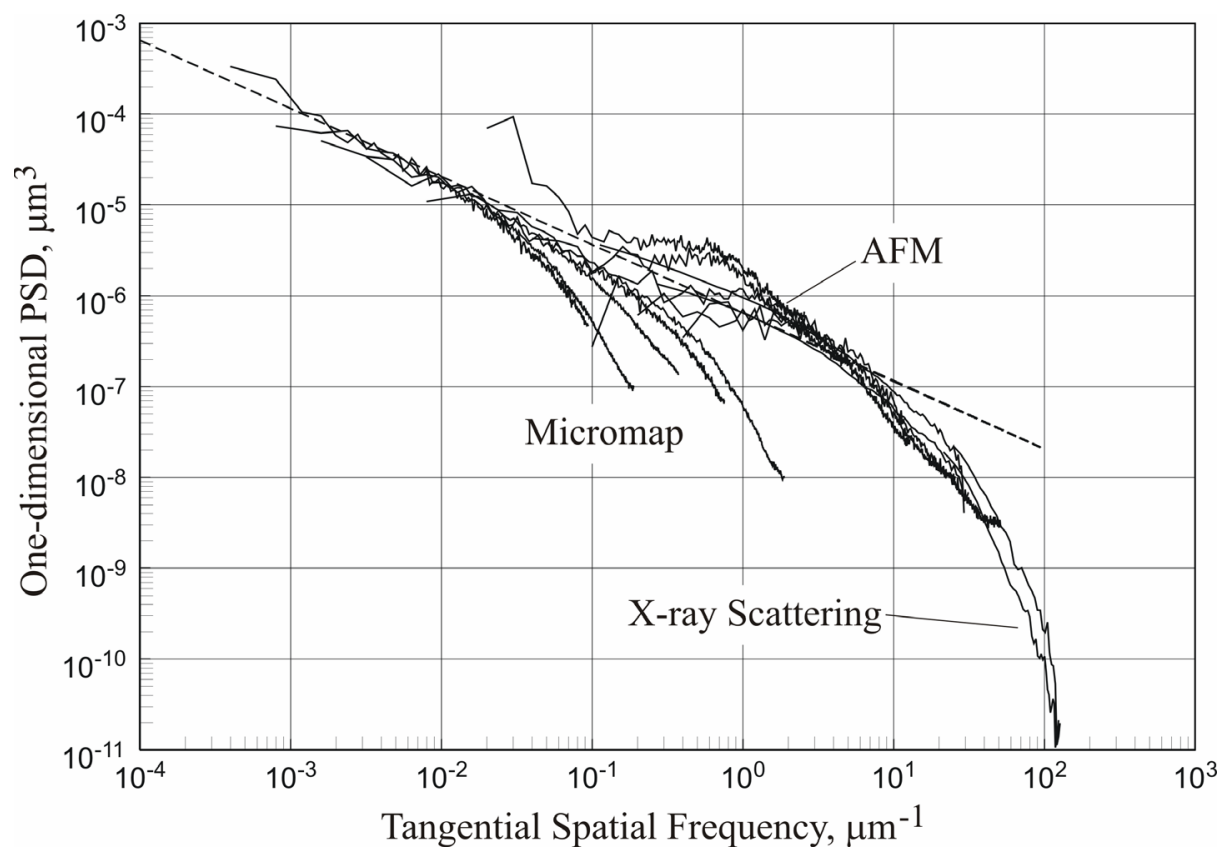

Figure 7: Tangential 1D PSD spectra obtained with the Micromap interferometric microscope, the Atomic Force Microscope, and the CXRO Reflectometry and Scattering experimental facility. The dashed line represents an inverse-power-law spectrum, enveloping all the measured spectra.

The main conclusion from the crosscheck is that these three techniques provide essentially consistent results. At all available spatial frequencies, the AFM measurements agree reasonably well with the X-ray scattering measurements. At the spatial frequencies approximately from $0.1 \mu \mathrm{m}^{-1}$ to $1 \mu \mathrm{m}^{-1}$, the AFM and X-ray scattering measurements can be thought as an extension of the Micromap measurements, but with higher spatial resolution and, therefore, free of the roll-off characteristic for the Micromap.

For the mirror under investigation, an inverse-power-law topography gives a good approximation for the measured

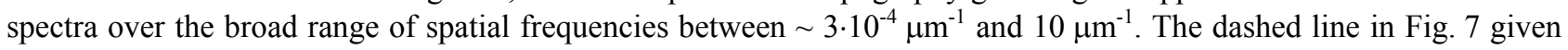
by the expression

$$
S_{1}\left(f_{x}\right)=S_{1}(1)\left(f_{x}\right)^{-\gamma} ; S_{1}(1) \cong 6.5 \cdot 10^{-7}, \gamma \cong 0.75
$$

represents such a power-law spectrum enveloping the measured spectra. In (6), the tangential spatial frequency $f_{x}$ and the tangential 1D PSD function $S_{1}\left(f_{x}\right)$ have dimensions of $\mu \mathrm{m}^{-1}$ and $\mu \mathrm{m}^{3}$, respectively. Due to the high degree of isotropy of the mirror surface finish, the approximated 1D PSD spectrum can be converted to a 2D PSD distribution by using a simple analytical expression. ${ }^{22}$ The resulted 2D PSD distribution can be used for correction of the high frequency roll-off characteristic for the Micromap measurements. The correction procedure developed in the present work is discussed in detail in Section 6.

\section{ESTIMATION OF THE MICROMAP MODULATION TRANSFER FUNCTION}

Most of the practical methods used to determine the 2D MTF function of an interferometric microscope are based on the PSD measurement of a 1D test surface, such as a step height standard, ${ }^{12,13}$ or a surface with isotropic finish as in the present work. In general, without additional assumptions, it is impossible to recover the 2D MTF just from 
measured 1D PSD spectra. The assumption about symmetry (isotropy) of the instrumental spatial resolution allows one to reduce the problem to searching for an effective 1D MTF. ${ }^{17}$

For a surface with isotropic topography, the 2D PSD distribution can be calculated from the known 1D PSD spectrum (see, e.g. ${ }^{4,22}$ ):

$$
S_{2}(f)=-\frac{1}{2 \pi} \int_{f}^{\infty} \frac{1}{\sqrt{f_{x}^{2}-f^{2}}} \frac{S_{1}\left(f_{x}\right)}{d f_{x}} d f_{x} ; \quad f^{2}=f_{x}^{2}+f_{y}^{2} .
$$

In the case of an isotropic inverse-power-law surface described by (6), the solution of (7) is given by

$$
S_{2}\left(f_{x}, f_{y}\right)=\frac{\Gamma[(\gamma+1) / 2] S_{1}(1)}{2 \sqrt{\pi} \Gamma[\gamma / 2]\left(f_{x}^{2}+f_{y}^{2}\right)^{(\gamma+1) / 2}},
$$

where $f_{x}$ and $f_{y}$ are the tangential and sagittal spatial frequencies, measured in $\mu \mathrm{m}^{-1}$. For the stainless steel mirror under investigation, the values of Gamma function are $\Gamma[(\gamma+1) / 2] \cong 2.37$ and $\Gamma[\gamma / 2] \cong 1.09$.

The procedure used to find the Micromap MTF consists of a few steps. First, the Micromap CCD camera read-out asymmetry is corrected based on the procedure described in detail elsewhere. ${ }^{17}$ Second, the inverse-power-law approximation of the 1D PSD function (6) is found and converted with equation (8) into an analytical expression for an isotropic 2D PSD distribution. The resulting analytical 2D PSD function is used to build a 2D PSD matrix of the theoretical, instrumentally unperturbed mirror surface. The theoretical 2D PSD matrix has the same size as the experimental (measured) PSD distribution that is $640 \times 480$ elements. Third, a correction matrix equal to the ratio of the measured and the theoretical PSDs is calculated. The square root of the matrix is the measured MTF, which, in principle, can be used to correct the Micromap PSD measurements with other mirrors. However, the measured 2D MTF contains the original spread of the experimental points and, therefore, will perturb the next measurements when correcting. In order to avoid this problem, in the fourth stage we sum the measured 2D PSD into the tangential and sagittal measured squared 1D MTFs. Comparison of the squared 1D MTFs shown in Fig. 8 gives one more possibility to insure the correctness of the procedure so far. The similarity of the squared 1D MTFs confirms the validity of the assumption about isotropy of the instrumental spatial resolution. In the next, fifth, stage, an analytical function best fitted to the spectrum averaged over two squared 1D MTFs in Fig. 8 is found and converted with equation (7) into the expected squared 2D MTF. This 2D distribution can be used for correction of the measured PSD distribution, which is smooth and free of the data spread perturbation mentioned above.

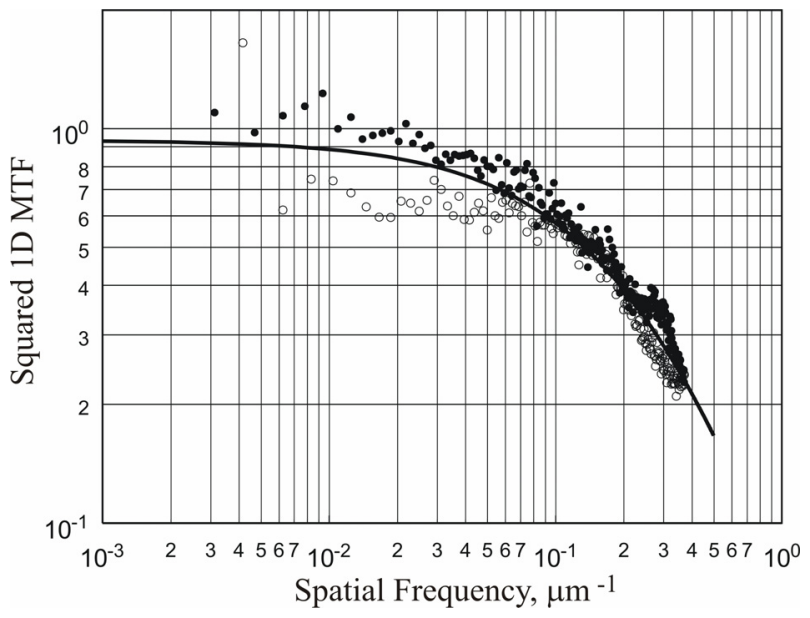

Figure 8: Tangential (filled circles) and sagittal (open circles) measured squared 1D MTFs of the Micromap interferometric microscope with the $10 \times$ objective. The solid line represents an analytical function best fitted to the spectrum averaged over two measured 1D MTFs. The function is $\propto(1+a f)^{-2}$ determined by a single parameter of the width, $a \approx 2.73 \mu \mathrm{m}$. This function with the width found and normalized to be equal to one at lower spatial frequencies was used to calculate the squared 2D MTF distribution by using expression (7).

Figure 9 illustrates efficiency of the developed MTF correction procedure applied to the PSD of the stainless steel mirror measured with the Micromap. Plots 1 in Fig. 9 are the tangential and sagittal 1D PSD spectra obtained with the $10 \times$ objective and shown among other Micromap spectra in Fig. 1a. The spectra are the result of application of the CCD 
read-out asymmetry correction; ${ }^{17}$ however, they still have the perturbation due to the uncorrected isotropic instrumental MTF. The plots 2 in Fig. 9 are obtained from the 2D PSD distribution corrected with the MTF determined in the way described in this section. The main result is that the procedure extends the spatial frequency range of the reliable PSD data via correction of a significant part of the high frequency roll-off clearly seen in plots 1 .

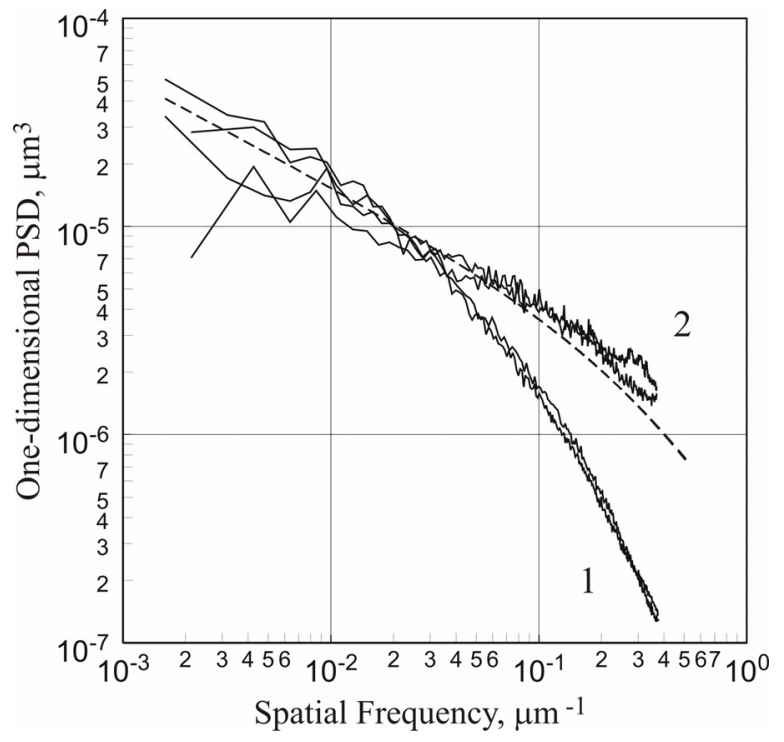

Figure 9: Tangential and sagittal 1D PSD spectra extracted from the Micromap measurement with the 10× objective. The stainless steel mirror used throughout the present work was measured. 1 - The original Micromap data were just processed to correct the CCD read-out asymmetry. ${ }^{17} 2$ - Tangential and sagittal 1D PSD spectra obtained after the correction procedure developed to account the instrumental MTF was applied to the same measured 2D PSD distribution. The dashed line represents the 1D spectrum calculated from the 2D inverse-power-law distribution (8) with parameters (6) via summing over the bandwidth, corresponding to the Micromap measurements with the $10 \times$ objective. Note the high frequency roll-off of the 1D power-law spectrum, which is due to the upper limit for the frequencies involved in the sum determined by the instrument bandwidth.

In spite of the fact that all illustrations are based on 1D PSD spectra (Figs. 8 and 9), the correction procedure was developed to correct the measured 2D PSD distributions. The corrected 2D PSD distributions ensure more reliable surface characterization and 3D calculations of X-ray scattering by a mirror.

\section{DISCUSSION AND CONCLUSIONS}

A remarkable observation of the present work is that an inverse-power-law behavior of the resulting1D PSD spectra of the stainless steel mirror under investigation serves as an envelope over the 1D PSD distributions measured with different techniques. A similar inverse-power-law behavior of 1D PSD spectra has been observed with X-ray mirrors made of silicon and glass-ceramic. ${ }^{5,21}$ In Refs. 5 and 21, the PSD measurements with a silicon mirror were performed with an interferometric microscope and a long trace profiler. The measurements were shown to be very consistent, covering the spatial frequency range from approximately $10^{-6} \mu \mathrm{m}^{-1}$ to $10^{-1} \mu \mathrm{m}^{-1}$. At this range of spatial frequencies, the composite spectrum of a cylindrical silicon mirror can be fit with two power law dependencies with power indexes $\gamma_{1}=1.871$ (at lower frequencies) and $\gamma_{2}=0.873$, crossing at frequency of approximately $5 \cdot 10^{-3} \mu \mathrm{m}^{-1}{ }^{23}$ Similar behavior of the PSD spectrum has been observed from a mirror with an aluminum substrate. ${ }^{23}$

We have also initiated an investigation of the PSD distribution of the stainless steel mirror at lower spatial frequencies. The investigation was performed with the $\mathrm{LTP}^{15}$ and ZYGO GPI interferometer, ${ }^{16}$ both available at the ALS OML.

Figure 10 shows the tangential PSD spectrum measured with the LTP. The LTP slope trace used for PSD transformation is the result of eight measurements taken over the same area of the mirror. The measurements are split into two sets of four measurements each performed at two different orientations of the mirror with respect to the LTP. Each set of four slope traces obtained at the same orientation were averaged to decrease the contribution of random noise. Then, the averaged traces were combined to exclude asymmetrical systematic error. The rms slope variation of the final trace is $\sim 0.7 \mu \mathrm{rad}$. The applied procedure was directed to minimize the apparatus random noise and systematic errors. However, as it was found by comparison of the PSD spectra obtained from the processed trace and from a trace of a single unprocessed measurement, the applied procedure has not any noticeable effect on the PSD distribution, excepting a decrease by a factor of approximately 1.5 of the PSD values for a few lowest frequency points. The 
spectrum in Fig. 10 can be also fitted with inverse-power-law dependence with a power index of approximately 3 shown with a dashed line. The index is significantly larger than that of the envelope over the PSD spectra at higher frequencies obtained with three other techniques. Note that the highest frequency in the plot in Fig. 10 is determined by the step of the LTP slope trace measurement of $1 \mathrm{~mm}$ approximately equal to the laser beam size in tangential direction. However, a more valid high frequency cut-off of the spectra has to relate to the beam size in sagittal direction, which is about $5 \mathrm{~mm}$.

The ZYGO GPI measurements provide surface height distribution data, which can be transferred into a PSD spectrum over a spatial frequency range significantly overlapped with the PSD measured with the LTP. Figure 11 shows the tangential 1D PSD spectra extracted from the ZYGO GPI measurements with the stainless steel mirror. The PSD spectra are the results of transformation of the same height distributions but detrended in a different way. Plot 1 in Fig. 11 corresponds to detrending with piston and tilt; whereas, plot 2 is obtained by detrending with a second power polynomial function. In both cases, the shown spectra are the result of averaging over four measurements of two different parts of the mirror. The smooth dashed line in Fig. 11 depicts a 1D PSD spectrum of a height distribution of a tilted ideal flat surface normalized to fit most of the spectrum shown with plot 1 . The data can be considered as an illustration of a strong dependence of the PSD analysis on detrending of the corresponding height distribution. This dependence is less pronounced, if a PSD spectrum is described with a power index smaller than two.
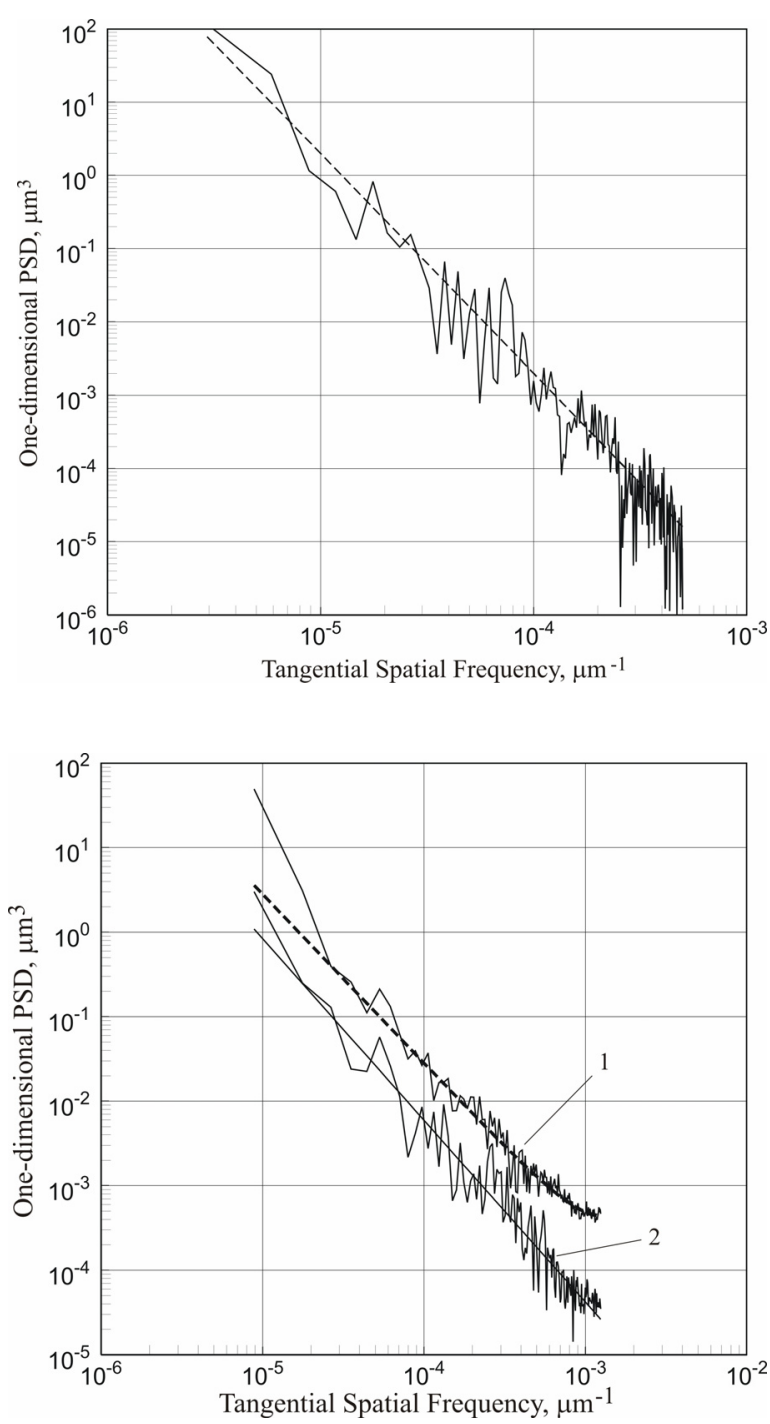

Figure 10: Tangential 1D PSD spectrum extracted from the LTP measurements with the stainless steel mirror. The spectrum corresponds to eight slope trace measurements combined in order to suppress the random noise and some systematic errors of the apparatus. However, the PSD spectrum for a single measurement was found to be indistinguishable from the spectrum shown here over almost the entire spatial frequency range. This suggests a relatively small contribution by noise and systematic errors. The LTP PSD spectrum has the inverse-power-law index that is approximately equal to three - the dashed line. Note that the PSD spectrum at higher frequencies measured with three other instruments discussed in this work can be enveloped over with an inverse-power-law curve determined by index of approximately 0.75 (see Sec. 6).

Figure 11: Tangential 1D PSD spectra extracted from the ZYGO GPI measurements with the stainless steel mirror. Each spectrum is the result of averaging over four measurements of two different parts of the mirror. 1 - Piston and tilt were detrended from the corresponding height distributions measured; 2 Detrending with a second power polynomial function was applied to the corresponding height distributions. The smooth dashed line reproduces the 1D PSD spectrum of a height distribution of a tilted ideal flat surface normalized to fit most of the spectrum linearly detrended. The data illustrate the dependence of the PSD analysis on detrending of the corresponding height distribution. The slope of the resulted PSD (plot 2), can be described with a power index of approximately 2.15 - the solid line. 
The PSD spectra measured with the LTP (Fig. 10) and with the interferometer (Fig. 11) are described with significantly different power index, equal to 3.0 and 2.15 , respectively. The most probable reason for the discrepancy is that in spite of all experimental precautions, and removal of some systematic error, the PSD spectra are still affected by residual instrumental noise and systematic error, which are comparable with the PSD distribution inherent in the mirror. The reliability of the measurements has to be confirmed with an additional investigation with significantly higher resolution. Just for references, figure 12 accumulates the tangential PSD spectra of the stainless steel mirror measured with all instruments.

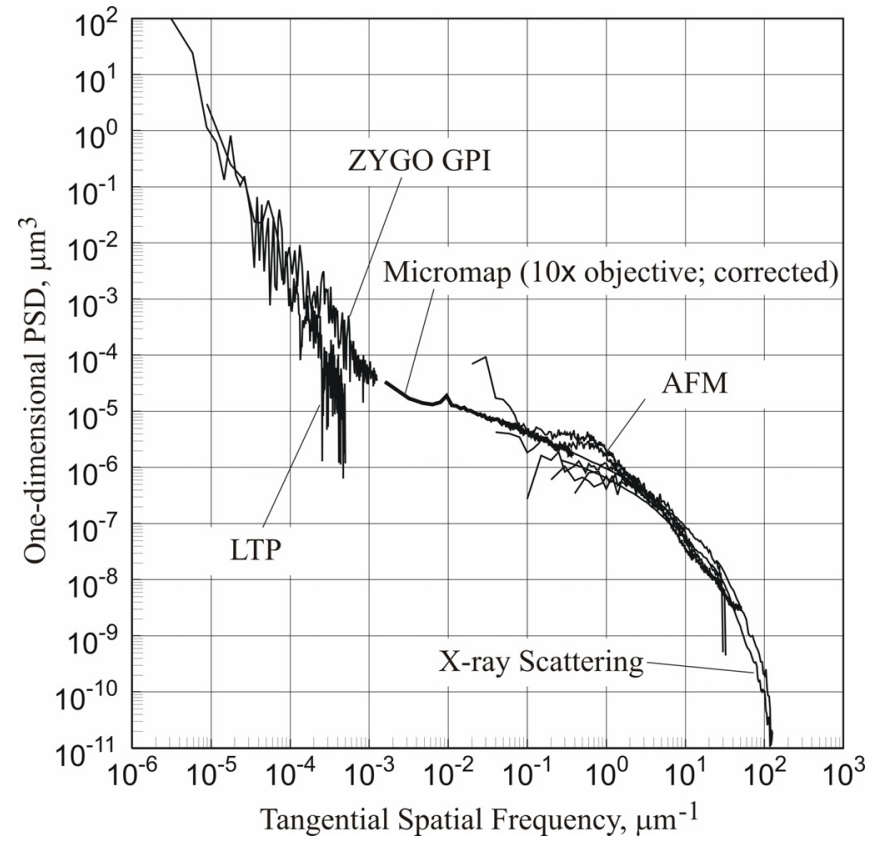

Figure 12: Tangential 1D PSD spectra obtained with the ZYGO GPI interferometer, the LTP, the Micromap interferometric microscope, the Atomic Force Microscope, and the CXRO Reflectometry and Scattering experimental facility (see comments in the text).
Besides the comprehensive comparison of the different techniques for measuring PSD distributions of surface roughness, a remarkable result of the present work is the procedure developed to account for the instrument MTF when measuring with the Micromap interferometric microscope. The procedure is based on the assumption about the isotropic character of the MTF. This assumption, proved experimentally - see Figure 8, became valid after the asymmetry of the Micromap CCD read-out was thoroughly corrected. ${ }^{17}$ Another crucial circumstance of the procedure is using a test mirror with an isotropic surface topology. This allows us to build an analytical correction function in the 2D domain based an inverse-power-law envelope over the Micromap, AFM, and X-ray scattering measurements.

The PSD measurement code with the correction procedure ${ }^{17}$ and accounting the instrument MTF was developed as a program which runs on the public domain IDL Virtual Machine. ${ }^{24}$ It is available upon request to our metrology lab for use, development, and beta testing. Once our testing is fully completed, it will be made available by web download for the use of the synchrotron optical and vendor communities via the ALS Optical Metrology Laboratory web-page. ${ }^{25}$ We fully expect that existing micro-roughness measuring instruments like the Micromap when combined with PSD analysis and Xray scattering calculations will remain essential tools for the metrology of a new generation of X-ray optics. In conclusion, we would like to refer to the work $^{26}$, which also provides a detailed investigation of different techniques for determining the rms roughness and PSDs of optical components.

\section{ACKNOWLEDGEMENTS}

The authors are grateful to Eugene Church, Howard Padmore, and Peter Takacs for helpful discussions, and Peter Kuschnir and Patrick Naulleau for their kind assistance with computation of the PSD of the Zygo data. The Advanced Light Source is supported by the Director, Office of Science, Office of Basic Energy Sciences, Materials Science Division, of the U.S. Department of Energy under Contract No. DE-AC02-05CH11231 at Lawrence Berkeley National Laboratory. 


\section{REFERENCES}

1. L. Assoufid, O. Hignette, M. Howells, S. Irick, H. Lammert, P. Takacs, Future metrology needs for synchrotron radiation grazing-incidence optics, Nucl. Instrum. and Meth. in Phys. Research A 467-468, 267-70 (2001).

2. E. L. Church, H. A. Jenkinson, and J. M. Zavada, Relationship between surface scattering and microtopographic features, Optical Engineering 18(2), 125-136 (1979).

3. D. Attwood, Soft X-rays and Extreme Ultraviolet Radiation (Cambridge University Press, New York, 1999).

4. E. L. Church, Fractal surface finish, Applied Optics 27(8), 1518-1526 (1988).

5. E. L. Church and P. Z. Takacs, Specification of surface figure and finish in terms of system performance, Applied Optics 32(19), 3344-3353 (1993).

6. R. N. Bracewell, The Fourier Transform and Its Applications (McGraw-Hill Publishing Company, New York, 1986), pp.108-112.

7. W. H. Press, S. A. Teukolsky, W. T. Vetterling, B. P. Flannery, Numerical Recipes in C++. The Art of Scientific Computing (Second edition; Cambridge University Press, New York, 2002).

8. Micromap Corporation (Tucson, AZ; phone +1 520-881-1911; fax +1 520-881-1913).

9. Digital Instruments/Veeco Instruments Inc., http://www.veeco.com/

10. http://www.cxro.lbl.gov/als6.3.2/

11. The mirror was fabricated by InSync, Inc. (Albuquerque, NM; http://www.insyncoptics.com) according to LBNL specifications.

12. G. D. Boreman, Modulation Transfer Function in Optical and Electro-optical Systems (SPIE Press, 2001).

13. P. Z. Takacs, A step height standard for surface profile characterization, Proceedings of SPIE, 1993 (1993), 65-74.

14. C. R. Wolfe, J. D. Downie, and J. K. Lawson, Measuring the spatial frequency transfer function of phasemeasuring interferometers for laser optics, Proceedings of SPIE, 2870 (1996), 553-7.

15. The LTP available in the ALS OML belongs to the second generation of the LTP systems, LTP II. The main difference between the LTP II and an instrument of the first generation [P. Z. Takacs, S-N. Qian, J. Colbert, Design of a long trace surface profiler, Proceedings of SPIE 749 (1987), 59-64] is the existence of a reference arm in addition to the measurement arm [S. C. Irick, W. R. McKinney, D. L. Lunt, P. Z. Takacs, Using a straightness reference in obtaining more accurate surface profiles from a long trace profiler (for synchrotron optics), Rev. Sci. Instrum., 63(1), 1436-8 (1992)].

16. ZYGO corporation (Laurel Brook Road, Middlefield, CT; http://www.zygo.com).

17. V. V. Yashchuk, A. D. Franck, S. C. Irick, M. R. Howells, A. A. MacDowell, W. R. McKinney, Two dimensional power spectral density measurements of X-ray optics with the Micromap interferometric microscope, SPIE Symposium on Optical Metrology 2005, part of LASER 2005, World of Photonics (Munich, Germany, 12-17 June 2005), Proceedings of SPIE Vol. 5858 (SPIE, Bellingham, WA, 2005) in press.

18. J. V. Bixler, C. W. Mauche, C. J. Hailey, and L. Medison, Reflectivity and scattering measurements of an Advanced X-ray Astrophysics Facility test coating sample, Applied Optics 34(28), 6542-6551 (1995).

19. V. E. Asadchikov， I. V. Kozhevnikov，Yu. S. Krivonosov， R. Mercier， T. H. Metzger， C. Morawe， E. Ziegler, Application of X-ray scattering technique to the study of supersmooth surfaces, Nuclear Instrum. and Methods in Physics Research A530, 575-595 (2004).

20. V. E. Asadchikov, A. Duparre, S. Jakobs, A. Yu. Karabekov, I. V. Kozhevnikov, Yu. S. Krivonosov, Comparative study of the roughness of optical surfaces and thin film by use of X-ray scattering and atomic force microscopy, Applied Optics 38(4), 684-691 (1999).

21. P. Z. Takacs, and E. L. Church, Figure and finish of grazing-incidence mirrors, Nuclear Instrum. and Methods in Physics Research A291, 253-264 (1990).

22. J. C. Stover, Optical Scattering: Measurement and Analysis (Second edition; SPIE Optical Engineering Press, Bellingham, 1995).

23. E. L. Church, and P. Z. Takacs, private communications.

24. http://www.rsinc.com/idl.

25. http://www-esg.lbl.gov/Production/OML.

26. A. Duparre, J. Ferre-Borrull, S. Gliech, G. Notni, J. Steinert, and J. M. Bennett, Surface characterization techniques for determining the root-mean-square roughness and power spectral densities of optical components, Applied Optics 41(1), 154-171 (2002).

*vvyashchuk@lbl.gov; phone +1 510-495-2592; fax +1 510-486-7696. 